\title{
Measurements of $\Lambda_{c}^{+}$branching fractions of Cabibbo-suppressed decay modes involving $\Lambda$ and $\Sigma^{0}$
}

B. Aubert, ${ }^{1}$ R. Barate, ${ }^{1}$ D. Boutigny, ${ }^{1}$ F. Couderc,${ }^{1}$ Y. Karyotakis,${ }^{1}$ J. P. Lees, ${ }^{1}$ V. Poireau,${ }^{1}$ V. Tisserand,${ }^{1}$ A. Zghiche, ${ }^{1}$ E. Grauges, ${ }^{2}$ A. Palano, ${ }^{3}$ M. Pappagallo, ${ }^{3}$ A. Pompili, ${ }^{3}$ J. C. Chen,${ }^{4}$ N. D. Qi,${ }^{4}$ G. Rong, ${ }^{4}$ P. Wang, ${ }^{4}$ Y. S. Zhu, ${ }^{4}$ G. Eigen, ${ }^{5}$ I. Ofte, ${ }^{5}$ B. Stugu, ${ }^{5}$ G. S. Abrams, ${ }^{6}$ M. Battaglia, ${ }^{6}$ D. Best,${ }^{6}$ A. B. Breon, ${ }^{6}$ D. N. Brown, ${ }^{6}$ J. Button-Shafer, ${ }^{6}$ R. N. Cahn, ${ }^{6}$

E. Charles, ${ }^{6}$ C. T. Day, ${ }^{6}$ M. S. Gill, ${ }^{6}$ A. V. Gritsan, ${ }^{6}$ Y. Groysman, ${ }^{6}$ R. G. Jacobsen, ${ }^{6}$ R. W. Kadel, ${ }^{6}$ J. Kadyk, ${ }^{6}$ L. T. Kerth, ${ }^{6}$ Yu. G. Kolomensky, ${ }^{6}$ G. Kukartsev, ${ }^{6}$ G. Lynch, ${ }^{6}$ L. M. Mir, ${ }^{6}$ P. J. Oddone, ${ }^{6}$ T. J. Orimoto, ${ }^{6}$ M. Pripstein, ${ }^{6}$ N. A. Roe, ${ }^{6}$

M. T. Ronan, ${ }^{6}$ W. A. Wenzel, ${ }^{6}$ M. Barrett, ${ }^{7}$ K. E. Ford, ${ }^{7}$ T. J. Harrison, ${ }^{7}$ A. J. Hart, ${ }^{7}$ C. M. Hawkes, ${ }^{7}$ S. E. Morgan, ${ }^{7}$

A. T. Watson, ${ }^{7}$ M. Fritsch,${ }^{8}$ K. Goetzen, ${ }^{8}$ T. Held,${ }^{8}$ H. Koch, ${ }^{8}$ B. Lewandowski, ${ }^{8}$ M. Pelizaeus, ${ }^{8}$ K. Peters,${ }^{8}$ T. Schroeder,${ }^{8}$

M. Steinke,${ }^{8}$ J. T. Boyd,${ }^{9}$ J. P. Burke, ${ }^{9}$ W. N. Cottingham, ${ }^{9}$ T. Cuhadar-Donszelmann, ${ }^{10}$ B. G. Fulsom, ${ }^{10}$ C. Hearty, ${ }^{10}$

N. S. Knecht,${ }^{10}$ T. S. Mattison, ${ }^{10}$ J. A. McKenna ${ }^{10}$ A. Khan, ${ }^{11}$ P. Kyberd, ${ }^{11}$ M. Saleem, ${ }^{11}$ L. Teodorescu, ${ }^{11}$ A. E. Blinov, ${ }^{12}$ V. E. Blinov, ${ }^{12}$ A. D. Bukin, ${ }^{12}$ V. P. Druzhinin, ${ }^{12}$ V. B. Golubev, ${ }^{12}$ E. A. Kravchenko, ${ }^{12}$ A. P. Onuchin, ${ }^{12}$ S. I. Serednyakov, ${ }^{12}$ Yu. I. Skovpen, ${ }^{12}$ E. P. Solodov, ${ }^{12}$ A. N. Yushkov, ${ }^{12}$ M. Bondioli, ${ }^{13}$ M. Bruinsma, ${ }^{13}$ M. Chao, ${ }^{13}$ S. Curry,${ }^{13}$ I. Eschrich, ${ }^{13}$ D. Kirkby, ${ }^{13}$ A. J. Lankford, ${ }^{13}$ P. Lund, ${ }^{13}$ M. Mandelkern, ${ }^{13}$ R. K. Mommsen, ${ }^{13}$ W. Roethel, ${ }^{13}$ D. P. Stoker, ${ }^{13}$ C. Buchanan, ${ }^{14}$ B. L. Hartfiel, ${ }^{14}$ S. D. Foulkes, ${ }^{15}$ J. W. Gary,${ }^{15}$ O. Long, ${ }^{15}$ B. C. Shen, ${ }^{15}$ K. Wang, ${ }^{15}$ L. Zhang, ${ }^{15}$ D. del Re, ${ }^{16}$ H. K. Hadavand, ${ }^{16}$ E. J. Hill, ${ }^{16}$ D. B. MacFarlane, ${ }^{16}$ H. P. Paar, ${ }^{16}$ S. Rahatlou, ${ }^{16}$ V. Sharma, ${ }^{16}$ J. W. Berryhill, ${ }^{17}$ C. Campagnari, ${ }^{17}$ A. Cunha, ${ }^{17}$ B. Dahmes,${ }^{17}$ T. M. Hong, ${ }^{17}$ M. A. Mazur, ${ }^{17}$ J. D. Richman, ${ }^{17}$ W. Verkerke, ${ }^{17}$ T. W. Beck, ${ }^{18}$ A. M. Eisner, ${ }^{18}$ C. J. Flacco, ${ }^{18}$ C. A. Heusch, ${ }^{18}$ J. Kroseberg, ${ }^{18}$ W. S. Lockman, ${ }^{18}$ G. Nesom, ${ }^{18}$ T. Schalk, ${ }^{18}$

B. A. Schumm, ${ }^{18}$ A. Seiden, ${ }^{18}$ P. Spradlin, ${ }^{18}$ D. C. Williams, ${ }^{18}$ M. G. Wilson, ${ }^{18}$ J. Albert, ${ }^{19}$ E. Chen, ${ }^{19}$

G. P. Dubois-Felsmann, ${ }^{19}$ A. Dvoretskii, ${ }^{19}$ D. G. Hitlin, ${ }^{19}$ J. S. Minamora, ${ }^{19}$ I. Narsky, ${ }^{19}$ T. Piatenko, ${ }^{19}$ F. C. Porter, ${ }^{19}$ A. Ryd ${ }^{19}$ A. Samuel, ${ }^{19}$ R. Andreassen, ${ }^{20}$ G. Mancinelli, ${ }^{20}$ B. T. Meadows,${ }^{20}$ M. D. Sokoloff,${ }^{20}$ F. Blanc, ${ }^{21}$ P. C. Bloom, ${ }^{21}$ S. Chen, ${ }^{21}$ W. T. Ford,${ }^{21}$ J. F. Hirschauer, ${ }^{21}$ A. Kreisel, ${ }^{21}$ U. Nauenberg, ${ }^{21}$ A. Olivas, ${ }^{21}$ W. O. Ruddick, ${ }^{21}$ J. G. Smith, ${ }^{21}$ K. A. Ulmer, ${ }^{21}$ S. R. Wagner, ${ }^{21}$ J. Zhang, ${ }^{21}$ A. Chen, ${ }^{22}$ E. A. Eckhart, ${ }^{22}$ A. Soffer, ${ }^{22}$ W. H. Toki, ${ }^{22}$ R. J. Wilson, ${ }^{22}$

F. Winklmeier, ${ }^{22}$ Q. Zeng, ${ }^{22}$ D. Altenburg, ${ }^{23}$ E. Feltresi, ${ }^{23}$ A. Hauke,${ }^{23}$ B. Spaan, ${ }^{23}$ T. Brandt,${ }^{24}$ J. Brose,${ }^{24}$ M. Dickopp,${ }^{24}$ V. Klose, ${ }^{24}$ H. M. Lacker, ${ }^{24}$ R. Nogowski, ${ }^{24}$ S. Otto, ${ }^{24}$ A. Petzold, ${ }^{24}$ J. Schubert, ${ }^{24}$ K. R. Schubert, ${ }^{24}$ R. Schwierz, ${ }^{24}$

J. E. Sundermann, ${ }^{24}$ D. Bernard, ${ }^{25}$ G. R. Bonneaud, ${ }^{25}$ P. Grenier, ${ }^{25}$ E. Latour, ${ }^{25}$ S. Schrenk,${ }^{25}$ Ch. Thiebaux, ${ }^{25}$

G. Vasileiadis, ${ }^{25}$ M. Verderi, ${ }^{25}$ D. J. Bard, ${ }^{26}$ P. J. Clark, ${ }^{26}$ W. Gradl, ${ }^{26}$ F. Muheim, ${ }^{26}$ S. Playfer, ${ }^{26}$ Y. Xie, ${ }^{26}$ M. Andreotti, ${ }^{27}$

D. Bettoni, ${ }^{27}$ C. Bozzi,${ }^{27}$ R. Calabrese,${ }^{27}$ G. Cibinetto, ${ }^{27}$ E. Luppi, ${ }^{27}$ M. Negrini, ${ }^{27}$ L. Piemontese, ${ }^{27}$ F. Anulli, ${ }^{28}$

R. Baldini-Ferroli, ${ }^{28}$ A. Calcaterra ${ }^{28}$ R. de Sangro, ${ }^{28}$ G. Finocchiaro, ${ }^{28}$ P. Patteri, ${ }^{28}$ I. M. Peruzzi, ${ }^{28, *}$ M. Piccolo, ${ }^{28}$ A. Zallo, ${ }^{28}$ A. Buzzo, ${ }^{29}$ R. Capra, ${ }^{29}$ R. Contri,${ }^{29}$ M. Lo Vetere, ${ }^{29}$ M. M. Macri ${ }^{29}$ M. R. Monge, ${ }^{29}$ S. Passaggio, ${ }^{29}$ C. Patrignani, ${ }^{29}$ E. Robutti, ${ }^{29}$ A. Santroni, ${ }^{29}$ S. Tosi, ${ }^{29}$ G. Brandenburg, ${ }^{30}$ K. S. Chaisanguanthum,${ }^{30}$ M. Morii, ${ }^{30}$ J. Wu, ${ }^{30}$ R. S. Dubitzky, ${ }^{31}$ U. Langenegger, ${ }^{31}$ J. Marks, ${ }^{31}$ S. Schenk, ${ }^{31}$ U. Uwer, ${ }^{31}$ W. Bhimji, ${ }^{32}$ D. A. Bowerman, ${ }^{32}$ P. D. Dauncey, ${ }^{32}$

U. Egede, ${ }^{32}$ R. L. Flack, ${ }^{32}$ J. R. Gaillard, ${ }^{32}$ J . A. Nash, ${ }^{32}$ M. B. Nikolich, ${ }^{32}$ W. Panduro Vazquez, ${ }^{32}$ X. Chai, ${ }^{33}$ M. J. Charles, ${ }^{33}$ W. F. Mader, ${ }^{33}$ U. Mallik, ${ }^{33}$ V. Ziegler, ${ }^{33}$ J. Cochran, ${ }^{34}$ H. B. Crawley, ${ }^{34}$ L. Dong, ${ }^{34}$ V. Eyges, ${ }^{34}$ W. T. Meyer, ${ }^{34}$ S. Prell, ${ }^{34}$ E. I. Rosenberg, ${ }^{34}$ A. E. Rubin, ${ }^{34}$ J. I. Yi, ${ }^{34}$ G. Schott,${ }^{35}$ N. Arnaud, ${ }^{36}$ M. Davier, ${ }^{36}$ X. Giroux,${ }^{36}$

G. Grosdidier, ${ }^{36}$ A. Höcker, ${ }^{36}$ F. Le Diberder, ${ }^{36}$ V. Lepeltier, ${ }^{36}$ A. M. Lutz, ${ }^{36}$ A. Oyanguren, ${ }^{36}$ T. C. Petersen, ${ }^{36}$

S. Plaszczynski, ${ }^{36}$ S. Rodier, ${ }^{36}$ P. Roudeau, ${ }^{36}$ M. H. Schune, ${ }^{36}$ A. Stocchi, ${ }^{36}$ W. F. Wang, ${ }^{36}$ G. Wormser, ${ }^{36}$ C. H. Cheng, ${ }^{37}$ D. J. Lange ${ }^{37}$ D. M. Wright, ${ }^{37}$ A. J. Bevan, ${ }^{38}$ C. A. Chavez, ${ }^{38}$ I. J. Forster, ${ }^{38}$ J. R. Fry,${ }^{38}$ E. Gabathuler, ${ }^{38}$ R. Gamet ${ }^{38}$ K. A. George, ${ }^{38}$ D. E. Hutchcroft,${ }^{38}$ R. J. Parry,${ }^{38}$ D. J. Payne, ${ }^{38}$ K. C. Schofield,${ }^{38}$ C. Touramanis,${ }^{38}$ F. Di Lodovico, ${ }^{39}$ W. Menges, ${ }^{39}$ R. Sacco, ${ }^{39}$ C. L. Brown, ${ }^{40}$ G. Cowan, ${ }^{40}$ H. U. Flaecher, ${ }^{40}$ M. G. Green, ${ }^{40}$ D. A. Hopkins, ${ }^{40}$ P. S. Jackson, ${ }^{40}$ T. R. McMahon, ${ }^{40}$ S. Ricciardi, ${ }^{40}$ F. Salvatore,${ }^{40}$ D. N. Brown, ${ }^{41}$ C. L. Davis, ${ }^{41}$ J. Allison, ${ }^{42}$ N. R. Barlow,${ }^{42}$ R. J. Barlow, ${ }^{42}$ Y. M. Chia, ${ }^{42}$ C. L. Edgar, ${ }^{42}$ M. C. Hodgkinson, ${ }^{42}$ M. P. Kelly, ${ }^{42}$ G. D. Lafferty, ${ }^{42}$ M. T. Naisbit, ${ }^{42}$ J. C. Williams, ${ }^{42}$ C. Chen ${ }^{43}$ W. D. Hulsbergen, ${ }^{43}$ A. Jawahery, ${ }^{43}$ D. Kovalskyi, ${ }^{43}$ C. K. Lae,${ }^{43}$ D. A. Roberts, ${ }^{43}$ G. Simi ${ }^{43}$ G. Blaylock,${ }^{44}$ C. Dallapiccola ${ }^{44}$ S. S. Hertzbach, ${ }^{44}$ R. Kofler,${ }^{44}$ X. Li,${ }^{44}$ T. B. Moore ${ }^{44}$ S. Saremi, ${ }^{44}$ H. Staengle,${ }^{44}$ S. Y. Willocq, ${ }^{44}$ R. Cowan, ${ }^{45}$ K. Koeneke, ${ }^{45}$ G. Sciolla, ${ }^{45}$ S. J. Sekula, ${ }^{45}$ M. Spitznagel, ${ }^{45}$ F. Taylor, ${ }^{45}$ R. K. Yamamoto, ${ }^{45}$ H. Kim, ${ }^{46}$

P. M. Patel ${ }^{46}$ S. H. Robertson, ${ }^{46}$ A. Lazzaro, ${ }^{47}$ V. Lombardo, ${ }^{47}$ F. Palombo, ${ }^{47}$ J. M. Bauer ${ }^{48}$ L. Cremaldi, ${ }^{48}$

V. Eschenburg, ${ }^{48}$ R. Godang, ${ }^{48}$ R. Kroeger ${ }^{48}$ J. Reidy, ${ }^{48}$ D. A. Sanders, ${ }^{48}$ D. J. Summers, ${ }^{48}$ H. W. Zhao, ${ }^{48}$ S. Brunet, ${ }^{49}$ D. Côté, ${ }^{49}$ P. Taras, ${ }^{49}$ F. B. Viaud, ${ }^{49}$ H. Nicholson, ${ }^{50}$ N. Cavallo, ${ }^{51, \dagger}$ G. De Nardo,${ }^{51}$ F. Fabozzi, ${ }^{51, \dagger}$ C. Gatto, ${ }^{51}$ L. Lista,${ }^{51}$ D. Monorchio, ${ }^{51}$ P. Paolucci, ${ }^{51}$ D. Piccolo,${ }^{51}$ C. Sciacca,${ }^{51}$ M. Baak,${ }^{52}$ H. Bulten,${ }^{52}$ G. Raven, ${ }^{52}$ H. L. Snoek, ${ }^{52}$ L. Wilden, ${ }^{52}$ C. P. Jessop, ${ }^{53}$ J. M. LoSecco, ${ }^{53}$ T. Allmendinger, ${ }^{54}$ G. Benelli, ${ }^{54}$ K. K. Gan,${ }^{54}$ K. Honscheid, ${ }^{54}$ D. Hufnagel, ${ }^{54}$ 
P. D. Jackson,${ }^{54}$ H. Kagan,${ }^{54}$ R. Kass,${ }^{54}$ T. Pulliam,${ }^{54}$ A. M. Rahimi,${ }^{54}$ R. Ter-Antonyan, ${ }^{54}$ Q. K. Wong, ${ }^{54}$ N. L. Blount,${ }^{55}$ J. Brau, ${ }^{55}$ R. Frey, ${ }^{55}$ O. Igonkina, ${ }^{55}$ M. Lu ${ }^{55}$ C. T. Potter, ${ }^{55}$ R. Rahmat,${ }^{55}$ N. B. Sinev,${ }^{55}$ D. Strom, ${ }^{55}$ J. Strube,${ }^{55}$ E. Torrence, ${ }^{55}$ F. Galeazzi, ${ }^{56}$ M. Margoni, ${ }^{56}$ M. Morandin, ${ }^{56}$ M. Posocco,${ }^{56}$ M. Rotondo,${ }^{56}$ F. Simonetto, ${ }^{56}$ R. Stroili, ${ }^{56}$ C. Voci, ${ }^{56}$ M. Benayoun, ${ }^{57}$ J. Chauveau,${ }^{57}$ P. David,${ }^{57}$ L. Del Buono,${ }^{57}$ Ch. de la Vaissière, ${ }^{57}$ O. Hamon, ${ }^{57}$ M. J. J. John, ${ }^{57}$ Ph. Leruste, ${ }^{57}$ J. Malclès,${ }^{57}$ J. Ocariz,${ }^{57}$ L. Roos,${ }^{57}$ G. Therin,${ }^{57}$ P. K. Behera,${ }^{58}$ L. Gladney, ${ }^{58}$ Q. H. Guo,${ }^{58}$ J. Panetta, ${ }^{58}$ M. Biasini,${ }^{59}$ R. Covarelli, ${ }^{59}$ S. Pacetti,${ }^{59}$ M. Pioppi, ${ }^{59}$ C. Angelini,${ }^{60}$ G. Batignani,${ }^{60}$ S. Bettarini,${ }^{60}$ F. Bucci,${ }^{60}$ G. Calderini, ${ }^{60}$ M. Carpinelli, ${ }^{60}$ R. Cenci, ${ }^{60}$ F. Forti, ${ }^{60}$ M. A. Giorgi, ${ }^{60}$ A. Lusiani, ${ }^{60}$ G. Marchiori, ${ }^{60}$ M. Morganti, ${ }^{60}$ N. Neri, ${ }^{60}$ E. Paoloni, ${ }^{60}$ M. Rama,${ }^{60}$ G. Rizzo, ${ }^{60}$ J. Walsh ${ }^{60}$ M. Haire,${ }^{61}$ D. Judd, ${ }^{61}$ D. E. Wagoner, ${ }^{61}$ J. Biesiada, ${ }^{62}$ N. Danielson, ${ }^{62}$ P. Elmer, ${ }^{62}$ Y. P. Lau, ${ }^{62}$ C. Lu, ${ }^{62}$ J. Olsen, ${ }^{62}$ A. J. S. Smith, ${ }^{62}$ A. V. Telnov, ${ }^{62}$ F. Bellini, ${ }^{63}$ G. Cavoto, ${ }^{63}$ A. D’ Orazio, ${ }^{63}$ E. Di Marco, ${ }^{63}$ R. Faccini, ${ }^{63}$ F. Ferrarotto, ${ }^{63}$ F. Ferroni, ${ }^{63}$ M. Gaspero, ${ }^{63}$ L. Li Gioi, ${ }^{63}$ M. A. Mazzoni, ${ }^{63}$ S. Morganti, ${ }^{63}$ G. Piredda,${ }^{63}$ F. Polci, ${ }^{63}$ F. Safai Tehrani, ${ }^{63}$ C. Voena, ${ }^{63}$ H. Schröder, ${ }^{64}$ R. Waldi, ${ }^{64}$ T. Adye,${ }^{65}$ N. De Groot, ${ }^{65}$ B. Franek, ${ }^{65}$ G. P. Gopal, ${ }^{65}$ E. O. Olaiya,${ }^{65}$ F. F. Wilson, ${ }^{65}$ R. Aleksan, ${ }^{66}$ S. Emery, ${ }^{66}$ A. Gaidot,${ }^{66}$ S. F. Ganzhur, ${ }^{66}$ G. Graziani, ${ }^{66}$ G. Hamel de Monchenault, ${ }^{66}$ W. Kozanecki, ${ }^{66}$ M. Legendre, ${ }^{66}$ G. W. London, ${ }^{66}$ B. Mayer,${ }^{66}$ G. Vasseur, ${ }^{66}$ Ch. Yèche, ${ }^{66}$ M. Zito, ${ }^{66}$ M. V. Purohit, ${ }^{67}$ A. W. Weidemann, ${ }^{67}$ J. R. Wilson, ${ }^{67}$ T. Abe, ${ }^{68}$ M. T. Allen,${ }^{68}$ D. Aston, ${ }^{68}$ R. Bartoldus, ${ }^{68}$ N. Berger ${ }^{68}$ A. M. Boyarski, ${ }^{68}$ O. L. Buchmueller,${ }^{68}$ R. Claus, ${ }^{68}$ J. P. Coleman, ${ }^{68}$ M. R. Convery, ${ }^{68}$ M. Cristinziani, ${ }^{68}$ J. C. Dingfelder, ${ }^{68}$ D. Dong, ${ }^{68}$ J. Dorfan ${ }^{68}$ D. Dujmic, ${ }^{68}$ W. Dunwoodie, ${ }^{68}$ S. Fan, ${ }^{68}$ R. C. Field, ${ }^{68}$ T. Glanzman, ${ }^{68}$ S. J. Gowdy, ${ }^{68}$ T. Hadig, ${ }^{68}$ V. Halyo, ${ }^{68}$ C. Hast, ${ }^{68}$ T. Hryn'ova, ${ }^{68}$ W. R. Innes,${ }^{68}$ M. H. Kelsey, ${ }^{68}$ P. Kim ${ }^{68}$ M. L. Kocian, ${ }^{68}$ D. W. G. S. Leith, ${ }^{68}$ J. Libby, ${ }^{68}$ S. Luitz ${ }^{68}$ V. Luth, ${ }^{68}$ H. L. Lynch, ${ }^{68}$ H. Marsiske, ${ }^{68}$ R. Messner, ${ }^{68}$ D. R. Muller, ${ }^{68}$ C. P. O' Grady, ${ }^{68}$ V. E. Ozcan, ${ }^{68}$ A. Perazzo,${ }^{68}$ M. Perl ${ }^{68}$ B. N. Ratcliff, ${ }^{68}$ A. Roodman, ${ }^{68}$ A. A. Salnikov, ${ }^{68}$ R. H. Schindler, ${ }^{68}$ J. Schwiening, ${ }^{68}$ A. Snyder, ${ }^{68}$ J. Stelzer, ${ }^{68}$ D. Su, ${ }^{68}$ M. K. Sullivan, ${ }^{68}$ K. Suzuki, ${ }^{68}$ S. K. Swain, ${ }^{68}$ J. M. Thompson, ${ }^{68}$ J. Va'vra, ${ }^{68}$ N. van Bakel, ${ }^{68}$ M. Weaver ${ }^{68}$ A. J. R. Weinstein, ${ }^{68}$ W. J. Wisniewski, ${ }^{68}$ M. Wittgen, ${ }^{68}$ D. H. Wright, ${ }^{68}$ A. K. Yarritu, ${ }^{68}$ K. Yi, ${ }^{68}$ C. C. Young, ${ }^{68}$ P. R. Burchat, ${ }^{69}$ A. J. Edwards, ${ }^{69}$ S. A. Majewski, ${ }^{69}$ B. A. Petersen, ${ }^{69}$ C. Roat, ${ }^{69}$ M. Ahmed, ${ }^{70}$ S. Ahmed,${ }^{70}$ M. S. Alam, ${ }^{70}$ R. Bula ${ }^{70}$ J. A. Ernst,${ }^{70}$ M. A. Saeed, ${ }^{70}$ F. R. Wappler, ${ }^{70}$ S. B. Zain, ${ }^{70}$ W. Bugg,${ }^{71}$ M. Krishnamurthy, ${ }^{71}$ S. M. Spanier, ${ }^{71}$ R. Eckmann, ${ }^{72}$ J. L. Ritchie, ${ }^{72}$ A. Satpathy,${ }^{72}$ R. F. Schwitters, ${ }^{72}$ J. M. Izen, ${ }^{73}$ I. Kitayama, ${ }^{73}$ X. C. Lou,${ }^{73}$ S. Ye,${ }^{73}$ F. Bianchi,${ }^{74}$ M. Bona,${ }^{74}$ F. Gallo, ${ }^{74}$ D. Gamba, ${ }^{74}$ M. Bomben, ${ }^{75}$ L. Bosisio, ${ }^{75}$ C. Cartaro, ${ }^{75}$ F. Cossutti, ${ }^{75}$ G. Della Ricca, ${ }^{75}$ S. Dittongo,${ }^{75}$ S. Grancagnolo, ${ }^{75}$ L. Lanceri ${ }^{75}$ L. Vitale, ${ }^{75}$ V. Azzolini ${ }^{76}$ F. Martinez-Vidal, ${ }^{76}$ R. S. Panvini,,${ }^{77}$ Sw. Banerjee, ${ }^{78}$ B. Bhuyan, ${ }^{78}$

C. M. Brown ${ }^{78}$ D. Fortin, ${ }^{78}$ K. Hamano,${ }^{78}$ R. Kowalewski, ${ }^{78}$ I. M. Nugent, ${ }^{78}$ J. M. Roney, ${ }^{78}$ R. J. Sobie, ${ }^{78}$ J. J. Back, ${ }^{79}$ P. F. Harrison, ${ }^{79}$ T. E. Latham, ${ }^{79}$ G. B. Mohanty, ${ }^{79}$ H. R. Band, ${ }^{80}$ X. Chen,${ }^{80}$ B. Cheng, ${ }^{80}$ S. Dasu, ${ }^{80}$ M. Datta, ${ }^{80}$ A. M. Eichenbaum, ${ }^{80}$ K. T. Flood,${ }^{80}$ M. T. Graham, ${ }^{80}$ J. J. Hollar, ${ }^{80}$ J. R. Johnson,${ }^{80}$ P. E. Kutter, ${ }^{80}$ H. Li, ${ }^{80}$ R. Liu, ${ }^{80}$ B. Mellado, ${ }^{80}$ A. Mihalyi, ${ }^{80}$ A. K. Mohapatra, ${ }^{80}$ Y. Pan, ${ }^{80}$ M. Pierini, ${ }^{80}$ R. Prepost,${ }^{80}$ P. Tan, ${ }^{80}$ S. L. Wu, ${ }^{80}$ Z. $\mathrm{Yu}^{80}$ and $\mathrm{H} . \mathrm{Neal}^{81}$

\title{
(BABAR Collaboration)
}

\author{
${ }^{1}$ Laboratoire de Physique des Particules, F-74941 Annecy-le-Vieux, France \\ ${ }^{2}$ IFAE, Universitat Autonoma de Barcelona, E-08193 Bellaterra, Barcelona, Spain \\ ${ }^{3}$ Università di Bari, Dipartimento di Fisica and INFN, I-70126 Bari, Italy \\ ${ }^{4}$ Institute of High Energy Physics, Beijing 100039, China \\ ${ }^{5}$ University of Bergen, Institute of Physics, N-5007 Bergen, Norway \\ ${ }^{6}$ Lawrence Berkeley National Laboratory and University of California, Berkeley, California 94720, USA \\ ${ }^{7}$ University of Birmingham, Birmingham, B15 2TT, United Kingdom \\ ${ }^{8}$ Ruhr Universität Bochum, Institut für Experimentalphysik 1, D-44780 Bochum, Germany \\ ${ }^{9}$ University of Bristol, Bristol BS8 1TL, United Kingdom \\ ${ }^{10}$ University of British Columbia, Vancouver, British Columbia, Canada V6T $1 \mathrm{Zl}$ \\ ${ }^{11}$ Brunel University, Uxbridge, Middlesex UB8 3PH, United Kingdom \\ ${ }^{12}$ Budker Institute of Nuclear Physics, Novosibirsk 630090, Russia \\ ${ }^{13}$ University of California at Irvine, Irvine, California 92697, USA \\ ${ }^{14}$ University of California at Los Angeles, Los Angeles, California 90024, USA \\ ${ }^{15}$ University of California at Riverside, Riverside, California 92521, USA \\ ${ }^{16}$ University of California at San Diego, La Jolla, California 92093, USA \\ ${ }^{17}$ University of California at Santa Barbara, Santa Barbara, California 93106, USA \\ ${ }^{18}$ University of California at Santa Cruz, Institute for Particle Physics, Santa Cruz, California 95064, USA \\ ${ }^{19}$ California Institute of Technology, Pasadena, California 91125, USA \\ ${ }^{20}$ University of Cincinnati, Cincinnati, Ohio 45221, USA
}




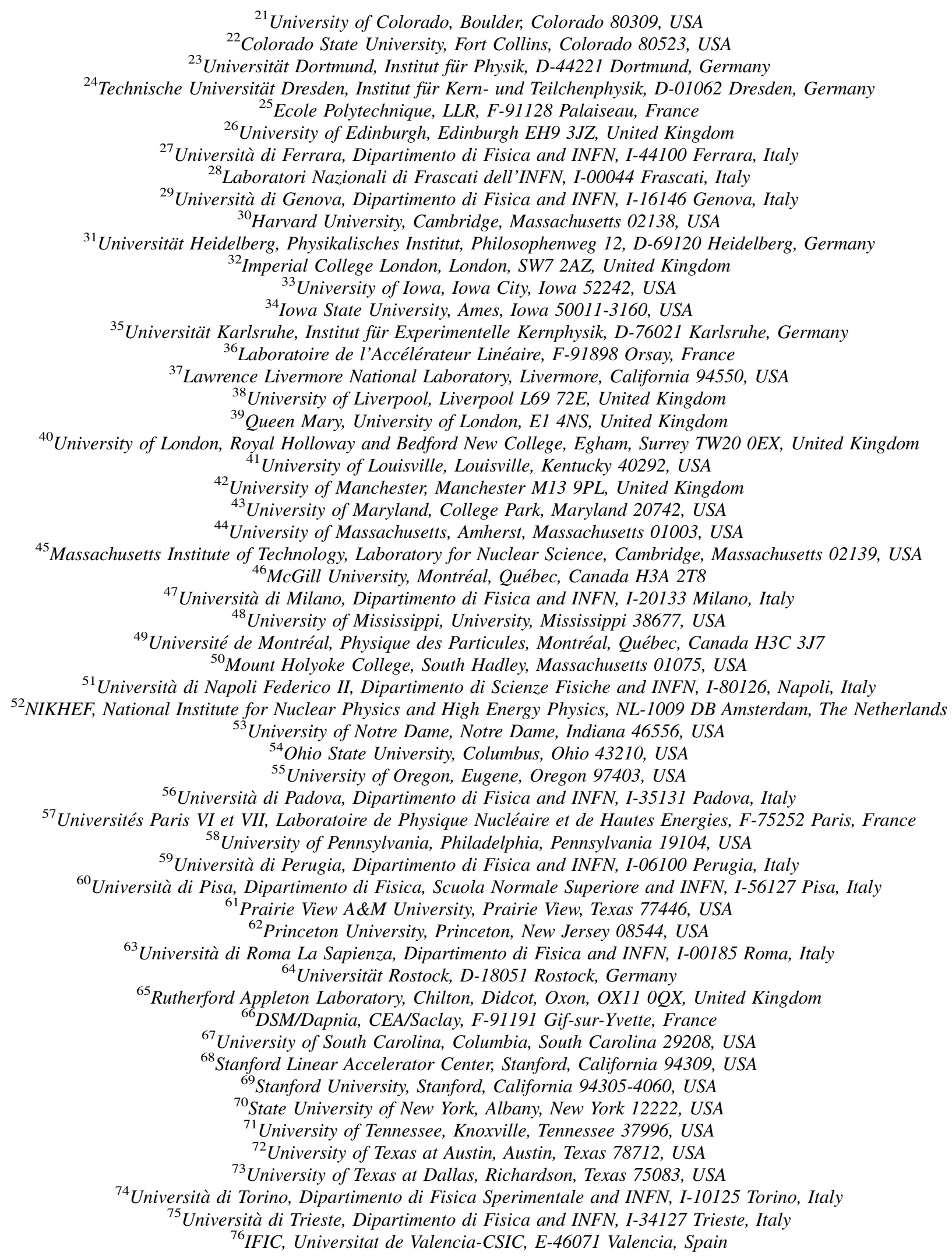

*Also at Università di Perugia, Dipartimento di Fisica, Perugia, Italy.

† Also at Università della Basilicata, Potenza, Italy.

ॠDeceased. 


\author{
${ }^{77}$ Vanderbilt University, Nashville, Tennessee 37235, USA \\ ${ }^{78}$ University of Victoria, Victoria, British Columbia, Canada V8W $3 P 6$ \\ ${ }^{79}$ Department of Physics, University of Warwick, Coventry CV4 7AL, United Kingdom \\ ${ }^{80}$ University of Wisconsin, Madison, Wisconsin 53706, USA \\ ${ }^{81}$ Yale University, New Haven, Connecticut 06511, USA
}

(Received 9 January 2006; revised manuscript received 28 January 2007; published 16 March 2007)

\begin{abstract}
We measure the branching ratios of the Cabibbo-suppressed decays $\Lambda_{c}^{+} \rightarrow \Lambda K^{+}$and $\Lambda_{c}^{+} \rightarrow \Sigma^{0} K^{+}$ relative to the Cabibbo-favored decay modes $\Lambda_{c}^{+} \rightarrow \Lambda \pi^{+}$and $\Lambda_{c}^{+} \rightarrow \Sigma^{0} \pi^{+}$to be $0.044 \pm 0.004$ (stat.) \pm 0.003 (syst.) and $0.038 \pm 0.005$ (stat.) \pm 0.003 (syst.), respectively. We set an upper limit on the branching ratio at the $90 \%$ confidence level for $\Lambda_{c}^{+} \rightarrow \Lambda K^{+} \pi^{+} \pi^{-}$of $4.1 \times 10^{-2}$ relative to $\Lambda_{c}^{+} \rightarrow \Lambda \pi^{+}$, and for $\Lambda_{c}^{+} \rightarrow \Sigma^{0} K^{+} \pi^{+} \pi^{-}$of $2.0 \times 10^{-2}$ relative to $\Lambda_{c}^{+} \rightarrow \Sigma^{0} \pi^{+}$. We also measure the branching fraction for the Cabibbo-favored mode $\Lambda_{c}^{+} \rightarrow \Sigma^{0} \pi^{+}$relative to $\Lambda_{c}^{+} \rightarrow \Lambda \pi^{+}$to be $0.977 \pm 0.015$ (stat.) \pm 0.051 (syst.). This analysis was performed using a data sample with an integrated luminosity of $125 \mathrm{fb}^{-1}$ collected by the $B A B A R$ detector at the PEP-II asymmetric-energy $B$ factory at SLAC.
\end{abstract}

DOI: 10.1103/PhysRevD.75.052002

PACS numbers: $13.30 .-\mathrm{a}$

\section{INTRODUCTION}

A considerable body of work has been done on charmed baryons [1]. Nonetheless, our understanding of the physics of charmed baryons has developed less rapidly than that of charmed mesons. This is due to the smaller baryon production cross sections, shorter lifetime, and, in $e^{+} e^{-}$storage rings, the absence of cleanly observable $\Lambda_{c} \bar{\Lambda}_{c}$ resonances. During the last few years there has been significant progress in the experimental study of the hadronic decays of charmed baryons. Recent results on masses, widths, lifetimes, production rates, and the decay asymmetry parameters of the charmed baryons have been published by different experiments; among them are the observations of Cabibbo-suppressed decays $\Lambda_{c}^{+} \rightarrow \mathrm{p} \phi$ by the CLEO Collaboration [2], and $\Lambda_{c}^{+} \rightarrow \Lambda K^{+}$and $\Lambda_{c}^{+} \rightarrow \Sigma^{0} K^{+}$by the Belle Collaboration [3].

The precision in the measurements of branching fractions is only about $40 \%$ for many Cabibbo-favored charm baryon modes [1], while for Cabibbo-suppressed decays the precision is even worse. As a consequence, we are not yet able to distinguish between the decay rate predictions made by different models, e.g., the quark model approach to nonleptonic charm decays $[4,5]$ and heavy quark effective theory [6]. Only one model [4] gives predictions for the Cabibbo-suppressed decays.

In this paper we present a study of $\Lambda_{c}^{+}$baryons produced in $e^{+} e^{-} \rightarrow q \bar{q}(q=u, d, s$, or $c$ quark) interactions at $B A B A R$. We present improved measurements of the Cabibbo-suppressed decays $\Lambda_{c}^{+} \rightarrow \Lambda K^{+}$and $\Lambda_{c}^{+} \rightarrow$ $\Sigma^{0} K^{+}$relative to Cabibbo-favored decays $\Lambda_{c}^{+} \rightarrow \Lambda \pi^{+}$ and $\Lambda_{c}^{+} \rightarrow \Sigma^{0} \pi^{+}$, respectively, and set an upper limit on the decay modes $\Lambda_{c}^{+} \rightarrow \Lambda K^{+} \pi^{+} \pi^{-}$, and $\Lambda_{c}^{+} \rightarrow$ $\Sigma^{0} K^{+} \pi^{+} \pi^{-}$relative to the same Cabibbo-favored decays. Here and throughout this paper, inclusion of chargeconjugate states is implied.

\section{THE BABAR DETECTOR AND DATA SAMPLES}

The data used in this analysis were collected by the $B A B A R$ detector at the PEP-II storage ring. We use data corresponding to a total integrated luminosity of $112 \mathrm{fb}^{-1}$ taken at the $Y(4 S)$ resonance (on-resonance) and $13 \mathrm{fb}^{-1}$ taken at a center-of-mass (CM) energy $40 \mathrm{MeV}$ below the $\Upsilon(4 S)$ mass and below the threshold of $B \bar{B}$ production (offresonance). A detailed description of the $B A B A R$ detector is presented in Ref. [7]. The components of the detector most relevant to this analysis are described here. Chargedparticle tracks are reconstructed with a five-layer, doublesided silicon vertex tracker (SVT) and a 40-layer drift chamber $(\mathrm{DCH})$ with a helium-based gas mixture, placed in a 1.5-T solenoidal field produced by a superconducting magnet. The resolution in $p_{T}$, the track momentum transverse to the beam direction, is approximately $\left(\delta p_{T} / p_{T}\right)^{2}=\left(0.0013(\mathrm{GeV} / c)^{-1} p_{T}\right)^{2}+(0.0045)^{2}$.

Kaons and protons are identified with likelihood ratios calculated from the ionization energy loss $(\mathrm{dE} / \mathrm{dx})$ measurements in the SVT and DCH, and from the observed pattern of Cherenkov light in an internally reflecting ring imaging detector (DIRC). The efficiency for identifying true kaons exceeds $80 \%$, while the probability for a pion to be misidentified as a kaon is less than $3 \%$. Photons are identified as isolated electromagnetic showers in a $\mathrm{CsI}(\mathrm{Tl})$ electromagnetic calorimeter. Large Monte Carlo (MC) samples generated with JETSET [8] are used to determine signal detection efficiency. The detector response in these samples is simulated with the GEANT4 [9] program. Particle identification efficiencies are corrected using data control samples.

\section{EVENT AND CANDIDATE SELECTION}

Candidates for $\Lambda$, which is in the final state of all the decay modes involved in this analysis, are reconstructed in the decay mode $\Lambda \rightarrow p \pi^{-}$. We fit the $p$ and $\pi^{-}$tracks to a common vertex and require the $\chi^{2}$ probability of the vertex fit to be greater than $0.1 \%$. The three-dimensional flight distance of each $\Lambda$ candidate between its decay vertex and the event primary vertex is required to be greater than $0.2 \mathrm{~cm}$.

This analysis is based on fits of invariant masses or differences between invariant masses, in the case of 
MEASUREMENTS OF $\Lambda_{c}^{+}$BRANCHING ...

$\Sigma^{0} \rightarrow \Lambda \gamma$ decays. In general, the fits are performed with the following criteria. For the signal, the sum of two Gaussian functions with a common mean (the two widths, the common mean and the fraction of the core Gaussian being free as discussed in detail in the corresponding fits below) is the preferred function to better reproduce the tails. However for the decay modes with less statistics, a single Gaussian with free mean and width is used. In case there is no statistically significant signal, a single Gaussian with fixed mean and width has to be used. The background is parametrized by a polynomial in invariant mass with order 2, or higher, as required to obtain a satisfactory fit.

The invariant mass of $\Lambda$ candidates is fitted using a sum of two Gaussian functions with a common mean to represent the signal and a 2nd order polynomial to represent the background. The fitted distribution is shown in Fig. 1. The resolution is measured to be $\sigma_{\mathrm{RMS}}=1.5 \mathrm{MeV} / \mathrm{c}^{2}$, where $\sigma_{\mathrm{RMS}}$ is defined by

$$
\sigma_{\mathrm{RMS}}^{2} \equiv f_{1} \sigma_{1}^{2}+f_{2} \sigma_{2}^{2},
$$

where $\sigma_{1}=0.820 \pm 0.003 \mathrm{MeV} / \mathrm{c}^{2}$ (the width of the core Gaussian) and $\sigma_{2}=2.103 \pm 0.021 \mathrm{MeV} / \mathrm{c}^{2}$ (the width of the wider Gaussian), and $f_{1}$ and $f_{2}\left(=1-f_{1}\right)$ are the two corresponding fractions of the two Gaussian functions, with $f_{2}=42 \%$ of candidates in the wider Gaussian. The mass of a $\Lambda$ candidate, used in the reconstruction of $\Lambda_{c}^{+}$or $\Sigma^{0}$ decays, is required to be in the range $1113 \mathrm{MeV} / c^{2}<$ $M_{p \pi^{-}}<1119 \mathrm{MeV} / c^{2}$.

The $\Sigma^{0}$ candidates are reconstructed in the decay mode $\Sigma^{0} \rightarrow \Lambda \gamma$ using the already selected $\Lambda$ sample and photons with an energy greater than $0.1 \mathrm{GeV}$. The mass difference $\left(M_{\gamma p \pi^{-}}-M_{p \pi^{-}}\right)$is shown in Fig. 2. The distribution is fit of the sum of two Gaussian functions with a common mean for the signal contribution, with $\sigma_{1}=2.00 \pm$ $0.18 \mathrm{MeV} / \mathrm{c}^{2}$ (the width of the core Gaussian) and $\sigma_{2}=$ $5.01 \pm 0.38 \mathrm{MeV} / \mathrm{c}^{2}$ (the width of the wider Gaussian). In

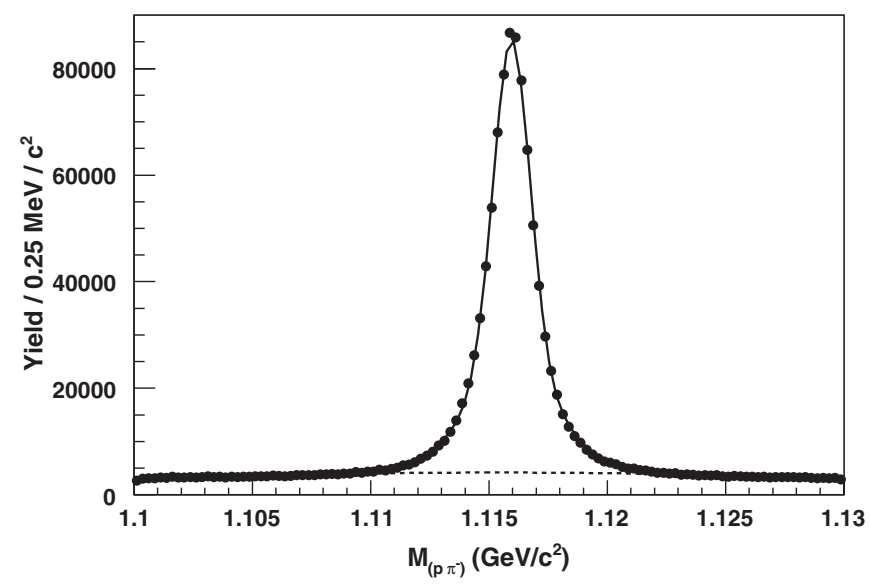

FIG. 1. The invariant mass of $p \pi^{-}$combinations. The solid line indicates the result of the fit for the sum of the signal and background and the dashed line for the background only.
PHYSICAL REVIEW D 75, 052002 (2007)

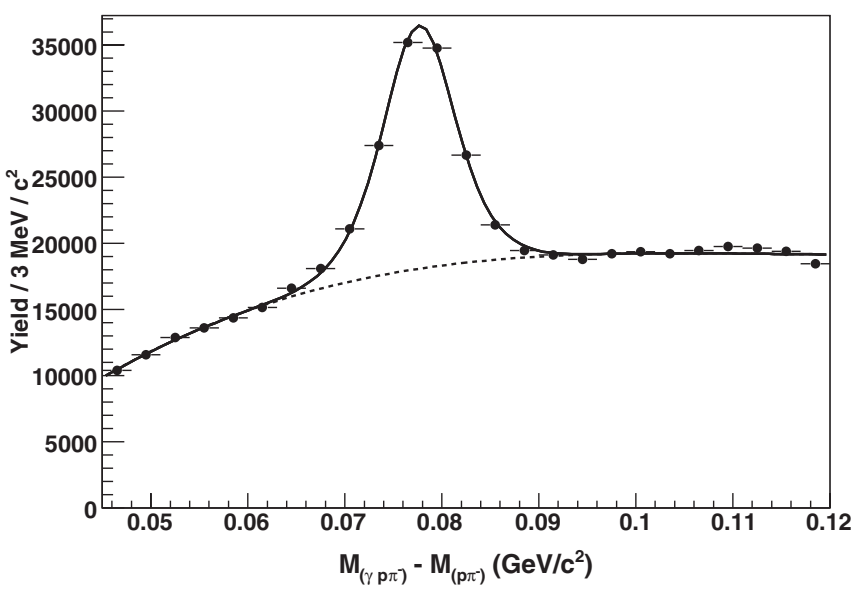

FIG. 2. The distribution of the invariant mass difference between $\gamma p \pi^{-}$combinations and $p \pi^{-}$candidates. The solid line indicates the result of the fit for the sum of the signal and background and the dashed line for the background only.

this fit $f_{2}=60 \%$, and a 3 rd order polynomial is used for the background. We obtain a resolution $\sigma_{\mathrm{RMS}}=$ $4.0 \mathrm{MeV} / \mathrm{c}^{2}$ and a mass difference between the $\Sigma^{0}$ and $\Lambda$ of $77.64 \pm 0.04$ (stat.) $\mathrm{MeV} / c^{2}$. We accept candidates with $\left(M_{\gamma p \pi^{-}}-M_{p \pi^{-}}\right)$within $10 \mathrm{MeV} / c^{2}$ of the mean value.

To suppress combinatorial and $B \bar{B}$ backgrounds, we introduce $x_{p}$ as a scaled momentum of a $\Lambda_{c}^{+}$candidate, where $x_{p}=p^{*} / p_{\max }^{*}$. Here $p^{*}$ is the reconstructed momentum of the $\Lambda_{c}^{+}$and $p_{\max }^{*}=\sqrt{s / 4-M^{2}}$ where $\sqrt{s}$ is the total CM energy and $M$ is the reconstructed mass of the $\Lambda_{c}^{+}$ candidate. Our search is limited to $x_{p}>0.5$ or $x_{p}>0.6$, depending on the decay mode so as to avoid the combinatorial background that dominates at low $x_{p}$.

\section{PHYSICS RESULTS}

\section{A. Study of the decays $\Lambda_{c}^{+} \rightarrow \Lambda K^{+}$and $\Lambda_{c}^{+} \rightarrow \Sigma^{0} K^{+}$}

The reconstructed $\Lambda$ candidates are combined with a $K^{+}$ with the requirement $x_{p}>0.5$ to produce the mass spectrum shown in Fig. 3. A clear $\Lambda_{c}^{+}$signal can be seen. The mass distribution is fitted using a Gaussian function for the signal, and a 2nd order polynomial for combinatorial background. The fit has a $\chi^{2}$ of 71.7 for 69 degrees of freedom. We obtain a raw yield of $1162 \pm 101$ (stat.) events and a fitted width $\sigma=5.5 \pm 0.7$ (stat.) $\mathrm{MeV} / c^{2}$, which is consistent with the resolution of $6.1 \pm 0.1 \mathrm{MeV} / c^{2}$ determined from a sample of simulated $\Lambda_{c}^{+} \rightarrow \Lambda K^{+}$signal events. The fitted mean value $2286.9 \pm 0.6 \mathrm{MeV} / c^{2}$ is found to be in agreement with the measured $\Lambda_{c}^{+}$mass $2286.46 \pm 0.14 \mathrm{MeV} / c^{2}[1]$.

For reference, we use the decay $\Lambda_{c}^{+} \rightarrow \Lambda \pi^{+}$. The invariant mass distribution of $\Lambda \pi^{+}$combinations is shown in Fig. 4. At mass values below the $\Lambda_{c}^{+}$mass a broad distribution around $2.2 \mathrm{GeV} / c^{2}$ is visible. This peak corre- 


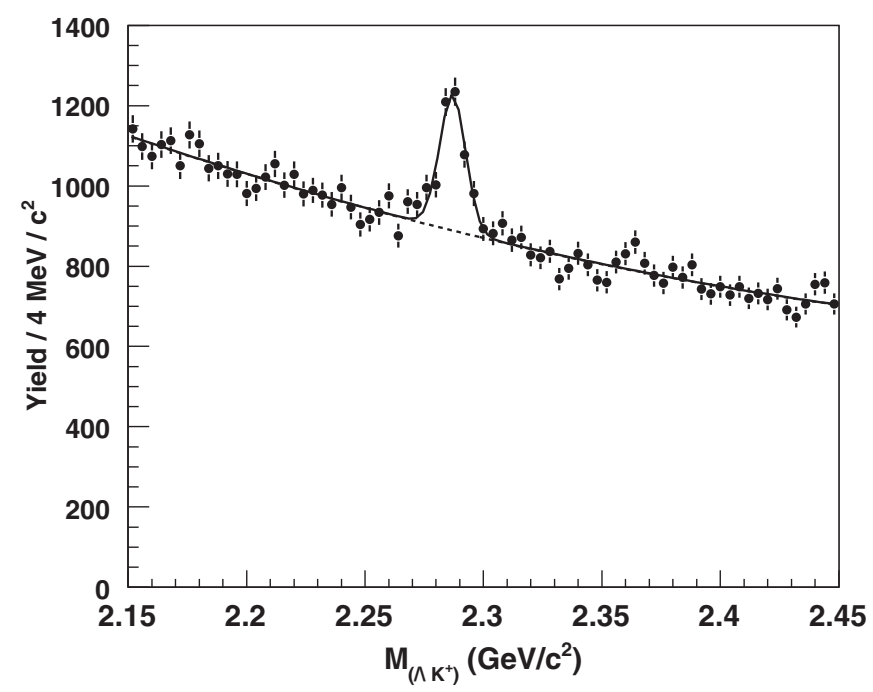

FIG. 3. The invariant mass of the $\Lambda K^{+}$combinations for $x_{p}>$ 0.5. The solid line indicates the result of the fit for the sum of the signal and background and the dashed line for the background only.

sponds to $\Lambda_{c}^{+} \rightarrow \Sigma^{0} \pi^{+}$with a missing $\gamma$. Additionally at $2.3 \mathrm{GeV} / c^{2}$ we see a shoulder, identified as the upper edge of a $\Xi_{c}^{+} / \Xi_{c}^{0}$ reflection with missing $\pi^{0} / \pi^{-}$. These shapes are established over the $\Lambda_{c}^{+}$signal region using a large sample of $q \bar{q}$ simulated events. The distribution is fitted using the sum of two Gaussian functions with the same mean for the signal, a square wave function for each reflection, and a 7 th order polynomial for combinatorial background. Because of the presence of reflections (as described above) in this decay mode, we need to use a

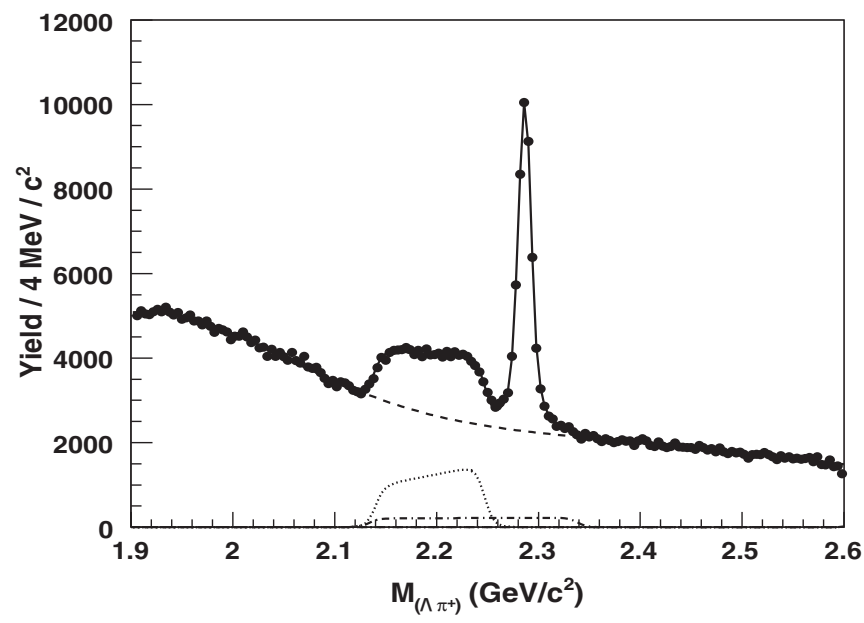

FIG. 4. The invariant mass of the $\Lambda \pi^{+}$combinations for $x_{p}>$ 0.5. The solid line indicates the result of the fit for the sum of the signal and backgrounds and the dashed line for the combinatorial background. The dotted line indicates the broad region corresponds to $\Lambda_{c}^{+} \rightarrow \Sigma^{0} \pi^{+}$with a missing $\gamma$ and the dashed-dotted line represents the region corresponding to $\Xi_{c}^{+} / \Xi_{c}^{0}$ reflection with missing $\pi^{0} / \pi^{-}$. wider window (1.9 to $2.6 \mathrm{GeV} / c^{2}$ instead of 2.15 to $2.45 \mathrm{GeV} / c^{2}$ ) to fit the background, and consequently a higher order polynomial to be able to reproduce the background shape over the extended range. The resultant $\chi^{2}$ of the fit to the data is 233.7 for 155 degrees of freedom. The fitted distribution gives a mean value of $2286.5 \pm$ $0.1 \mathrm{MeV} / c^{2}$ which is in agreement with the measured $\Lambda_{c}^{+}$mass [1]. The fitted values for the width of the core Gaussian and, for the width of the wider Gaussian are $\sigma_{1}=$ $5.6 \pm 0.1 \mathrm{MeV} / c^{2}$ and, $\sigma_{2}=11.6 \pm 0.3 \mathrm{MeV} / c^{2}$ respectively, with $f_{2}=36 \%$. We obtain a raw yield of $33543 \pm$ 334 (stat.) events with the measured resolution $\sigma_{\mathrm{RMS}}=$ $8.3 \pm 0.3$ (stat.) $\mathrm{MeV} / c^{2}$, which is consistent with the resolution of $8.0 \pm 0.1$ (stat.) $\mathrm{MeV} / \mathrm{c}^{2}$ measured from a sample of simulated $\Lambda_{c}^{+} \rightarrow \Lambda \pi^{+}$signal events. Using signal MC, the ratio of signal reconstruction efficiencies $\epsilon$ is found to be $\epsilon\left(\Lambda_{c}^{+} \rightarrow \Lambda K^{+}\right) / \epsilon\left(\Lambda_{c}^{+} \rightarrow \Lambda \pi^{+}\right)=0.781 \pm 0.010$ (stat.). With this value we calculate:

$$
\frac{\mathcal{B}\left(\Lambda_{\mathrm{c}}^{+} \rightarrow \Lambda \mathrm{K}^{+}\right)}{\mathcal{B}\left(\Lambda_{\mathrm{c}}^{+} \rightarrow \Lambda \pi^{+}\right)}=0.044 \pm 0.004 \text { (stat.) } \pm 0.003 \text { (syst.). }
$$

As a cross-check, we calculate the ratio $\mathcal{B}\left(\Lambda_{c}^{+} \rightarrow\right.$ $\left.\Lambda K^{+}\right) / \mathcal{B}\left(\Lambda_{c}^{+} \rightarrow \Lambda \pi^{+}\right)$in on-resonance and off-resonance data separately. The value obtained using on- and off-peak samples agree within uncertainties (the ratio of on-peak to off-peak branching ratio is: $1.04 \pm 0.04$ ). We provide a detailed description of the sources of systematic uncertainty in Sec. V.

We also use the invariant mass distribution of $\Lambda \pi^{+}$as shown in Fig. 4 to extract the yield of $\Lambda_{c}^{+} \rightarrow \Sigma^{0} \pi^{+}$assuming a missing $\gamma$. The signal yield which is extracted from the corresponding square wave function fit is found to be: $32693 \pm 324$. We generate signal MC samples of $\Lambda_{c}^{+} \rightarrow$ $\Sigma^{0} \pi^{+}$with missing $\gamma$ to evaluate the signal detection efficiency and to get the signal shape. The systematic uncertainty due to this is considered and is included as a part of the total systematic uncertainty for this branching ratio. The relative signal reconstruction efficiency from the $\mathrm{MC}$ is found to be $\epsilon\left(\Lambda_{c}^{+} \rightarrow \Sigma^{0} \pi^{+}\right) / \epsilon\left(\Lambda_{c}^{+} \rightarrow \Lambda \pi^{+}\right)=$ $1.013 \pm 0.010$ (stat.). We measure

$$
\left.\frac{\mathcal{B}\left(\Lambda_{\mathrm{c}}^{+} \rightarrow \Sigma^{0} \pi^{+}\right)}{\mathcal{B}\left(\Lambda_{\mathrm{c}}^{+} \rightarrow \Lambda \pi^{+}\right)}=0.977 \pm 0.015 \text { (stat.) } \pm 0.051 \text { (syst. }\right) \text {. }
$$

We combine the reconstructed $\Sigma^{0}$ candidates with a $K^{+}$ to form $\Lambda_{c}^{+}$candidates and require $x_{p}>0.5$. We improve the invariant mass resolution by about $20 \%$ by using the variable, $M_{\Sigma^{0} K^{+}}-M_{\Sigma^{0}}+M_{\Sigma^{0}}^{P D G}$, instead of $M_{\Sigma^{0} K^{+}}$, where $M_{\Sigma^{0}}$ is the reconstructed mass of the $\Sigma^{0}$ and $M_{\Sigma^{0}}^{P D G}$ is the world average for the mass of the $\Sigma^{0}$ [1]. This method is also used in other experiments to improve the mass resolution [3]. For demonstration purposes we also show, in Fig. 5, the $\Lambda_{c}^{+} \rightarrow \Sigma^{0} K^{+}$mass distribution, where we do not replace the mass of $\Sigma^{0}$ by the fixed mass (PDG) value. The fit uses a Gaussian for the signal and a 3rd order polynomial to represent the background. The fitted yield 
MEASUREMENTS OF $\Lambda_{c}^{+}$BRANCHING $\ldots$

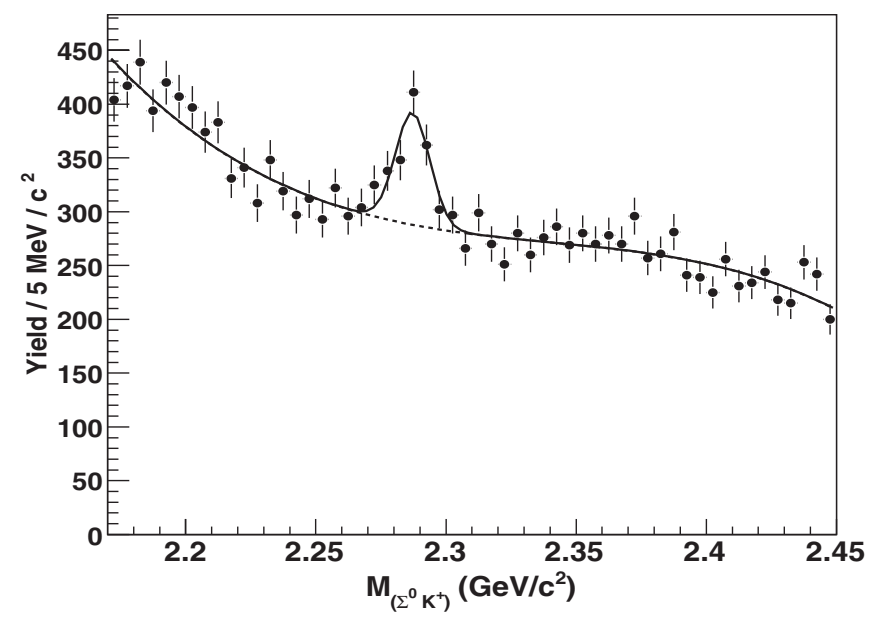

FIG. 5. The invariant mass of $\Sigma^{0}(\Lambda \gamma) K^{+}$combinations for $x_{p}>0.5$, where we do not replace the $\Sigma^{0}$ mass with the PDG value of the $\Sigma^{0}$ mass, as compared to what we have done for our final fit (shown in Fig. 6). The solid line indicates the result of the fit for the sum of the signal and background and the dashed line for the background only.

is $323 \pm 64$ (stat.) events with measured width of $\sigma=$ $6.1 \pm 1.5 \mathrm{MeV} / c^{2}$. This fit has a $\chi^{2}$ of 51.8 for 49 degrees of freedom. The final fit for the invariant mass distribution of $\Sigma^{0} K^{+}$combinations is shown in Fig. 6. An attempt to fit the $\Lambda_{c}^{+}$mass distribution to the sum of a single Gaussian and a 2 nd order polynomial shape yields a high $\chi^{2}$ of 72.4 for 50 degrees of freedom. However, if the fit is performed using a single Gaussian function for the signal and a 3rd order polynomial for combinatorial background, the resultant $\chi^{2}$ is 47.8 for 49 degrees of freedom. The fit yields $366 \pm 52$ (stat.) events. The measured width $\sigma=$ $5.7 \pm 0.8 \mathrm{MeV} / c^{2}$ is consistent with the resolution $\sigma=$ $6.0 \pm 0.1 \mathrm{MeV} / c^{2}$ determined from a sample of simulated

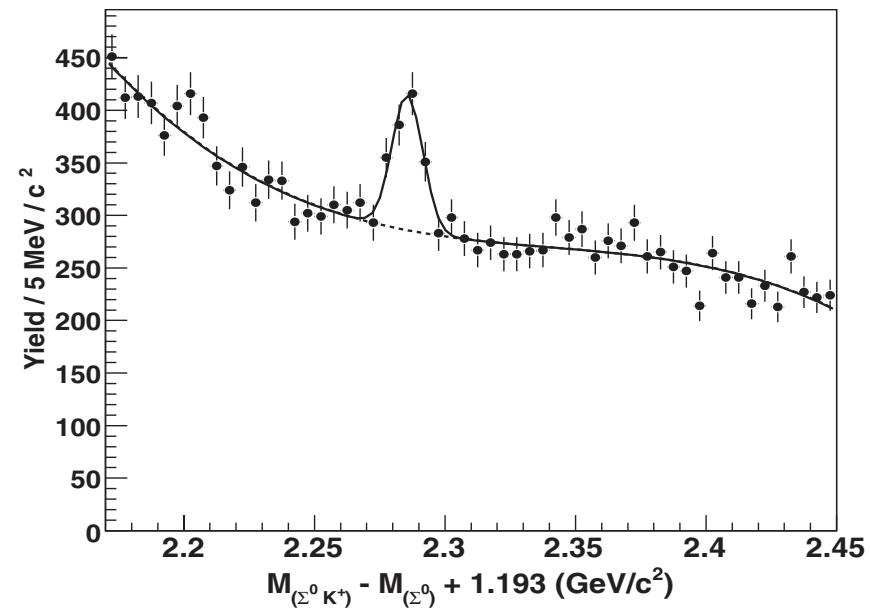

FIG. 6. The invariant mass of $\Sigma^{0}(\Lambda \gamma) K^{+}$combinations for $x_{p}>0.5$. The solid line indicates the result of the fit for the sum of the signal and background and the dashed line for the background only.
PHYSICAL REVIEW D 75, 052002 (2007)

$\Lambda_{c}^{+} \rightarrow \Sigma^{0} K^{+}$signal events. The fitted mean value $2286.0 \pm 0.9 \mathrm{MeV} / c^{2}$ is in agreement with the measured $\Lambda_{c}^{+} \operatorname{mass}[1]$.

For reference, we use the Cabibbo-favored decay mode $\Lambda_{c}^{+} \rightarrow \Sigma^{0} \pi^{+}$. The invariant mass of the $\Sigma^{0} \pi^{+}$combinations is shown in Fig. 7. An attempt to fit this distribution to a sum of single Gaussian and a 2nd order polynomial gives a $\chi^{2}$ of 119.9 for 54 degrees of freedom, which is not the best choice for this fit. However, the final fit uses a Gaussian function for the signal and a 3rd order polynomial for background, gives a $\chi^{2}$ of 87.3 for 53 degrees of freedom. The fit yields $12490 \pm 162$ (stat.) events. The measured width of $\sigma=6.7 \pm 0.1 \mathrm{MeV} / c^{2}$ is consistent with the resolution $\sigma=7.1 \pm 0.1 \mathrm{MeV} / c^{2}$ measured in a sample of simulated $\Lambda_{c}^{+} \rightarrow \Sigma^{0} \pi^{+}$signal events. The fitted mean value $2285.6 \pm 0.7 \mathrm{MeV} / c^{2}$ is also in agreement with the measured $\Lambda_{c}^{+}$mass [1]. The relative reconstruction efficiency is measured to be $\epsilon\left(\Lambda_{c}^{+} \rightarrow \Sigma^{0} K^{+}\right) / \epsilon\left(\Lambda_{c}^{+} \rightarrow \Sigma^{0} \pi^{+}\right)=$ $0.780 \pm 0.010$ (stat.) using signal MC samples. The resulting relative branching ratio is

$$
\frac{\mathcal{B}\left(\Lambda_{\mathrm{c}}^{+} \rightarrow \Sigma^{0} \mathrm{~K}^{+}\right)}{\mathcal{B}\left(\Lambda_{\mathrm{c}}^{+} \rightarrow \Sigma^{0} \pi^{+}\right)}=0.038 \pm 0.005 \text { (stat.) } \pm 0.003 \text { (syst.) }
$$

\section{B. Search for the decay of $\Lambda_{c}^{+} \rightarrow \Lambda K^{+} \pi^{+} \pi^{-}$}

To measure the Cabibbo-suppressed decay $\Lambda_{c}^{+} \rightarrow$ $\Lambda K^{+} \pi^{+} \pi^{-}$we use the selection criteria described in Sec. III. This decay mode has multiple particles in the final state. The combinatorial background is relatively higher here than in the processes like $\Lambda_{c}^{+} \rightarrow \Lambda \pi^{+} / K^{+}$. The scaled momentum is restricted to $x_{p}>0.6$ in order to reduce the combinatorial background. For the reference we use the $\Lambda_{c}^{+} \rightarrow \Lambda \pi^{+}$decay mode with the same scaled momentum selection, for which we obtain a raw yield of $22204 \pm 257$ (stat.) events.

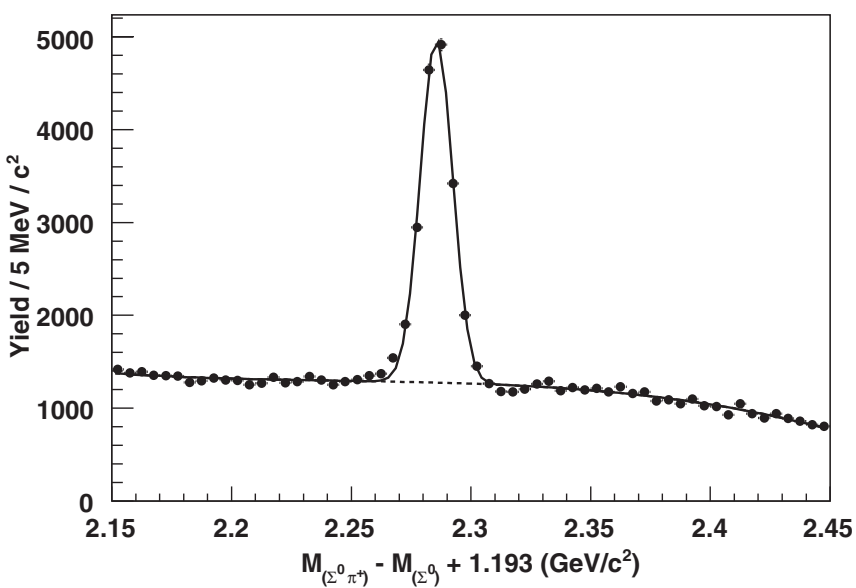

FIG. 7. The invariant mass of $\Sigma^{0}(\Lambda \gamma) \pi^{+}$combinations for $x_{p}>0.5$. The solid line indicates the result of the fit for the sum of the signal and background and the dashed line for the background only. 
We search for all the possible decays leading to the same final state as $\Lambda K^{+} \pi^{+} \pi^{-}$and find that the major contributions come from the decays of $\Lambda_{c}^{+} \rightarrow \Xi^{-} K^{+} \pi^{+}\left(\Xi^{-} \rightarrow\right.$ $\left.\Lambda \pi^{-}\right)$and $\Lambda_{c}^{+} \rightarrow \Lambda \mathrm{K}_{\mathrm{S}}^{0} K^{+}\left(\mathrm{K}_{\mathrm{S}}^{0} \rightarrow \pi^{+} \pi^{-}\right)$. We confirm these contributions using our $q \bar{q}$ simulated events. We find that each daughter particle in the decays of $\Lambda_{c}^{+} \rightarrow$ $\Xi^{-} K^{+} \pi^{+}$and $\Lambda_{c}^{+} \rightarrow \Lambda \mathrm{K}_{\mathrm{S}}^{0} K^{+}$, comes from the $\Lambda_{c}^{+}$signal. We reconstruct a $\Xi^{-}$candidate from a $\Lambda$ candidate and a $\pi^{-}$track requiring an invariant mass within $15 \mathrm{MeV} / c^{2}$ around the nominal value $1321.3 \mathrm{MeV} / c^{2}$ [1]. The invariant mass distribution of $\Xi^{-} K^{+} \pi^{+}$combinations is shown in Fig. 8. The distribution is fitted using a single Gaussian for the signal and a 2nd order polynomial for the background, with a resultant $\chi^{2}$ of 67.5 for 54 degrees of freedom. We obtain a width $\sigma=6.6 \pm 0.2 \mathrm{MeV} / c^{2}$ and a signal yield of $2665 \pm 84$ (stat.). The relative signal reconstruction efficiency is measured to be $\epsilon\left(\Lambda_{c}^{+} \rightarrow \Xi^{-} K^{+} \pi^{+}\right) / \epsilon\left(\Lambda_{c}^{+} \rightarrow \Lambda \pi^{+}\right)=0.250 \pm 0.003$ (stat.). Accounting for the $\Xi^{-}$subdecay branching fraction [1], the branching ratio is measured to be

$$
\begin{aligned}
\frac{\mathcal{B}\left(\Lambda_{\mathrm{c}}^{+} \rightarrow \Xi^{-} \mathrm{K}^{+} \pi^{+}\right)}{\mathcal{B}\left(\Lambda_{\mathrm{c}}^{+} \rightarrow \Lambda \pi^{+}\right)}= & 0.480 \pm 0.016 \text { (stat.) } \\
& \pm 0.038 \text { (syst.) }
\end{aligned}
$$

We also reconstruct $K_{S}^{0}$ candidates formed from two tracks identified as a $\pi^{+}$and a $\pi^{-}$with invariant mass $489<M_{\pi \pi}<509 \mathrm{MeV} / c^{2}$. The invariant mass distribution of $\Lambda_{c}^{+} \rightarrow \Lambda \mathrm{K}_{\mathrm{S}}^{0} K^{+}$is shown in Fig. 9. The fit is performed using a single Gaussian for the $\Lambda_{c}^{+}$signal whereas the background is described by a 2 nd order polynomial function. The resultant fit has a $\chi^{2}$ of 43.5 for 54 degrees of freedom. The measured width is $5.5 \pm$ 0.4 (stat.) $\mathrm{MeV} / \mathrm{c}^{2}$ and a signal yield of $460 \pm 30$ (stat.) is obtained. Using a signal reconstruction efficiency of $\epsilon\left(\Lambda_{c}^{+} \rightarrow \Lambda \mathrm{K}_{\mathrm{S}}^{0} K^{+}\right) / \epsilon\left(\Lambda_{c}^{+} \rightarrow \Lambda \pi^{+}\right)=0.152 \pm 0.020$ (stat.)

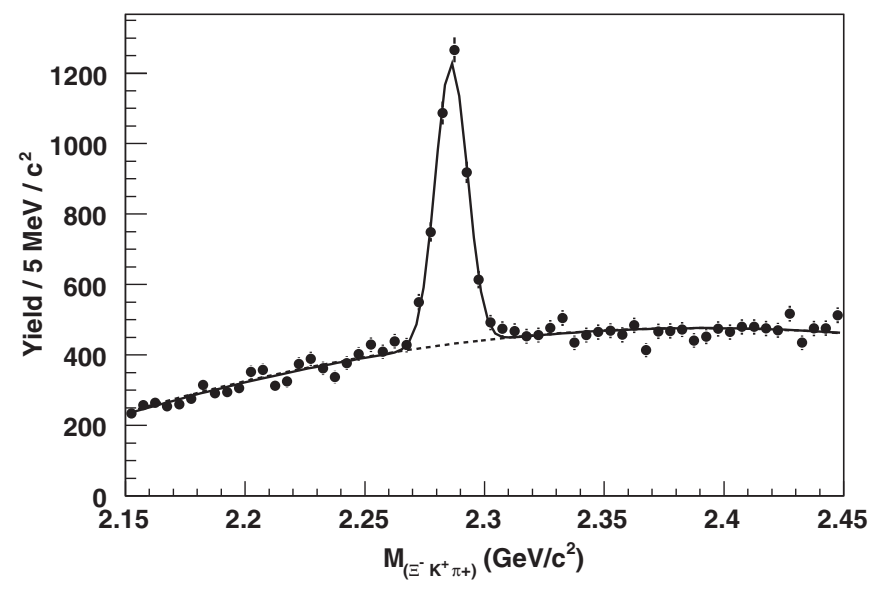

FIG. 8. Combinatorial $\Xi^{-}\left(\Lambda \pi^{-}\right) K^{+} \pi^{+}$invariant mass distribution for $x_{p}>0.6$. The solid line indicates the result of the fit for the sum of the signal and background and the dashed line for the background only.

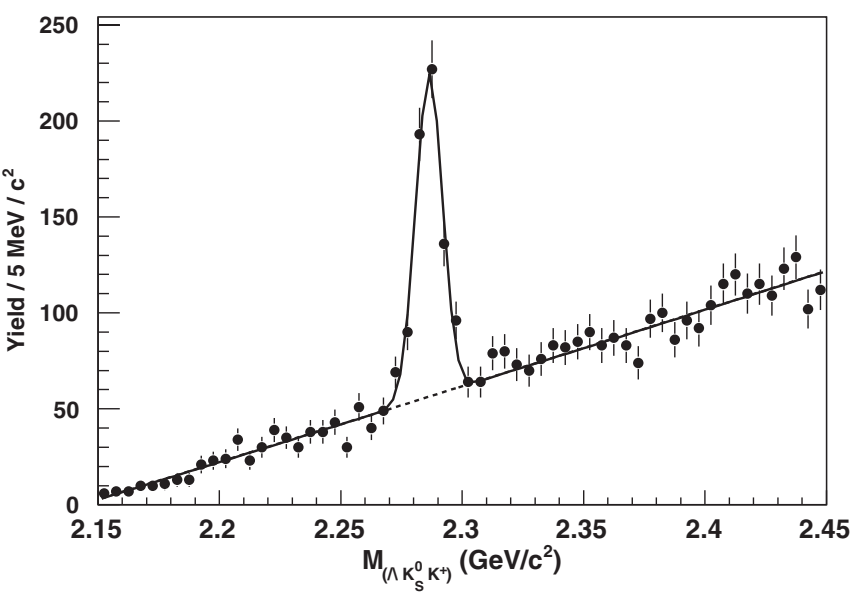

FIG. 9. Combinatorial $\Lambda \mathrm{K}_{\mathrm{S}}^{0}\left(\pi^{+} \pi^{-}\right) K^{+}$invariant mass distribution for $x_{p}>0.6$. The solid line indicates the result of the fit for the sum of the signal and background and the dashed line for the background only.

and accounting for the $\overline{\mathrm{K}}^{0}\left(\overline{\mathrm{K}}^{0} \rightarrow \mathrm{K}_{\mathrm{S}}^{0}\right)$ and $\mathrm{K}_{\mathrm{S}}^{0}\left(\mathrm{~K}_{\mathrm{S}}^{0} \rightarrow\right.$ $\pi^{+} \pi^{-}$) subdecay branching fractions [1], the branching ratio is measured to be

$$
\begin{aligned}
\frac{\mathcal{B}\left(\Lambda_{\mathrm{c}}^{+} \rightarrow \Lambda \overline{\mathrm{K}}^{0} \mathrm{~K}^{+}\right)}{\mathcal{B}\left(\Lambda_{\mathrm{c}}^{+} \rightarrow \Lambda \pi^{+}\right)}= & 0.395 \pm 0.026(\text { stat. }) \\
& \pm 0.036 \text { (syst. })
\end{aligned}
$$

We reject the contribution from the above Cabibbofavored decay modes by excluding the $\Xi^{-}$and $\mathrm{K}_{\mathrm{S}}^{0}$ mass windows as mentioned above. The final invariant mass distribution of $\Lambda K^{+} \pi^{+} \pi^{-}$combinations is shown in Fig. 10. We fit the mass distribution using a Gaussian function for the signal and a 2 nd order polynomial for the combinatorial background. We fix the width $\sigma=$ $5.2 \mathrm{MeV} / c^{2}$ and the mean $2285.5 \mathrm{MeV} / c^{2}$ as predicted

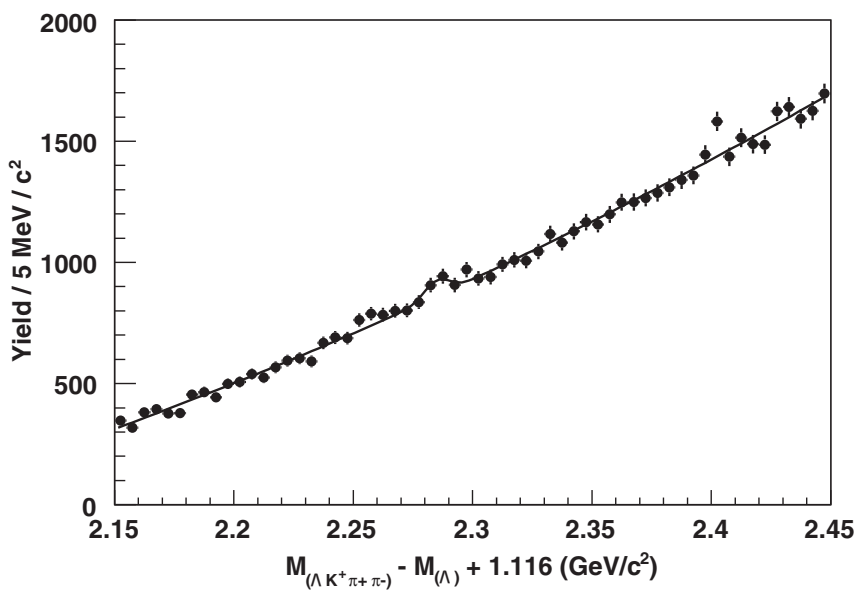

FIG. 10. The invariant mass of $\Lambda K^{+} \pi^{+} \pi^{-}$combinations for $x_{p}>0.6$. The solid line indicates the result of the fit for the sum of the signal and background. 
MEASUREMENTS OF $\Lambda_{c}^{+}$BRANCHING ...

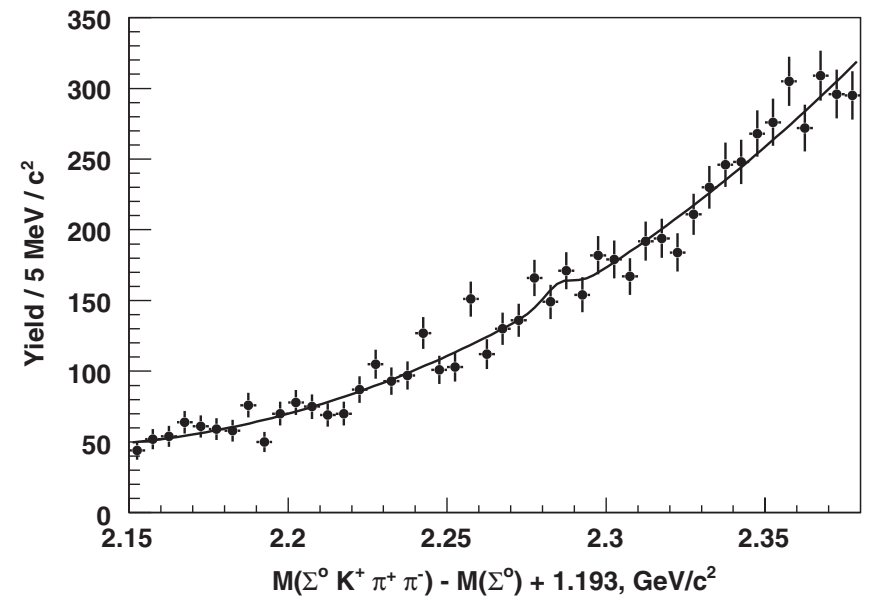

FIG. 11. The invariant mass of $\Sigma^{0}(\Lambda \gamma) K^{+} \pi^{+} \pi^{-}$combinations for $x_{p}>0.6$. The solid line indicates the result of the fit for the sum of the signal and background.

from a sample of simulated signal events for this decay. We obtain a signal yield of $158 \pm 63$ (stat.) events for the $\Lambda_{c}^{+} \rightarrow \Lambda K^{+} \pi^{+} \pi^{-}$decay. The goodness for this fit shows a $\chi^{2}$ of 56.6 for 56 degrees of freedom. The relative signal reconstruction efficiency is measured to be $\epsilon\left(\Lambda_{c}^{+} \rightarrow\right.$ $\left.\Lambda K^{+} \pi^{+} \pi^{-}\right) / \epsilon\left(\Lambda_{c}^{+} \rightarrow \Lambda \pi^{+}\right)=0.310 \pm 0.010$ (stat.).

Since we do not observe a statistically significant signal for $\Lambda_{c}^{+} \rightarrow \Lambda K^{+} \pi^{+} \pi^{-}$, we calculate the upper limit at $90 \%$ confidence level (C.L.) using the Feldman and Cousins method [10] and including systematic uncertainties.

$$
\frac{\mathcal{B}\left(\Lambda_{\mathrm{c}}^{+} \rightarrow \Lambda \mathrm{K}^{+} \pi^{+} \pi^{-}\right)}{\mathcal{B}\left(\Lambda_{\mathrm{c}}^{+} \rightarrow \Lambda \pi^{+}\right)}<4.1 \times 10^{-2} \text { at } 90 \% \text { C.L. }
$$

PHYSICAL REVIEW D 75, 052002 (2007)

\section{Search for the decay of $\Lambda_{c}^{+} \rightarrow \Sigma^{0} K^{+} \pi^{+} \pi^{-}$}

We search for the decay $\Lambda_{c}^{+} \rightarrow \Sigma^{0} K^{+} \pi^{+} \pi^{-}$using the selection described in Sec. III and restricting the scaled momentum to $x_{p}>0.6$. The invariant mass distribution of $\Sigma^{0} K^{+} \pi^{+} \pi^{-}$is shown in Fig. 11. The $\Lambda_{c}^{+}$mass distribution is fitted using a single Gaussian function with fixed width $\sigma=4.4 \mathrm{MeV} / c^{2}$ and mean $=2284.7 \mathrm{MeV} / c^{2}$ (measured from a sample of simulated signal events for this decay) for the signal and a 2nd order polynomial for combinatorial background, with a $\chi^{2}$ of 48.9 for 41 degrees of freedom. The fit yields $21 \pm 24$ (stat.) events. Using the decay mode $\Lambda_{c}^{+} \rightarrow \Sigma^{0} \pi^{+}$for reference, we find a raw yield of $8848 \pm$ 126(stat.) events for this decay in the range $x_{p}>0.6$. The relative reconstruction efficiency is determined to be $\epsilon\left(\Lambda_{c}^{+} \rightarrow \Sigma^{0} K^{+} \pi^{+} \pi^{-}\right) / \epsilon\left(\Lambda_{c}^{+} \rightarrow \Sigma^{0} \pi^{+}\right)=0.390 \pm$ 0.010 (stat.). We do not observe a statistically significant signal for $\Lambda_{c}^{+} \rightarrow \Sigma^{0} K^{+} \pi^{+} \pi^{-}$. We calculate the upper limit using the Feldman and Cousins method [10] and including systematic uncertainties. We find:

$$
\frac{\mathcal{B}\left(\Lambda_{\mathrm{c}}^{+} \rightarrow \Sigma^{0} \mathrm{~K}^{+} \pi^{+} \pi^{-}\right)}{\mathcal{B}\left(\Lambda_{\mathrm{c}}^{+} \rightarrow \Sigma^{0} \pi^{+}\right)}<2.0 \times 10^{-2} \text { at } 90 \% \text { C.L. }
$$

\section{SYSTEMATIC STUDIES}

We consider several possible sources of systematic uncertainties in our measurements, as shown in Table I. The systematic uncertainty due to limited signal MC statistics is between $1 \%$ and $3 \%$ depending on the decay mode. The systematic uncertainty due to each requirement in the candidate selection is estimated by varying the selection cuts (mass window for the resonance masses, cuts on $\Lambda$ flight distance and vertex $\chi^{2}, x_{p}$ cut). It is typically about $1 \%$ and always below $4 \%$. For $\mathcal{B}\left(\Lambda_{c}^{+} \rightarrow \Sigma^{0} K^{+}\right) / \mathcal{B}\left(\Lambda_{c}^{+} \rightarrow\right.$ $\left.\Sigma^{0} \pi^{+}\right)$, the photon spectrum is different in the signal and

\begin{tabular}{|c|c|c|c|c|c|c|c|}
\hline Sources of uncertainty & $\Lambda K^{+}$ & $\Lambda K^{+} \pi^{+} \pi^{-}$ & $\Sigma^{0} K^{+}$ & $\Sigma^{0} K^{+} \pi^{+} \pi^{-}$ & $\Sigma^{0} \pi^{+}$ & $\Xi^{-} K^{+} \pi^{+}$ & $\Lambda \bar{K}^{0} K^{+}$ \\
\hline MC statistics & 1.1 & 1.9 & 1.6 & 2.4 & $\ldots$ & 2.0 & 2.1 \\
\hline$\Lambda_{\text {mass }}$ & 0.6 & 0.1 & 1.2 & 1.2 & $\ldots$ & 1.1 & 0.1 \\
\hline$P_{\chi^{2}}$ & 3.8 & 0.7 & 0.9 & 0.9 & $\ldots$ & 0.7 & 0.7 \\
\hline$\Lambda$ flight & 0.7 & 2.8 & 1.9 & 1.9 & $\ldots$ & 2.4 & 3.4 \\
\hline$x_{p}$ & 0.7 & 1.8 & 2.1 & 2.1 & $\ldots$ & 2.2 & 1.8 \\
\hline$E_{\gamma}$ & $\ldots$ & $\ldots$ & 0.9 & 0.9 & $\ldots$ & $\ldots$ & $\ldots$ \\
\hline Tracking & $\ldots$ & 2.8 & $\ldots$ & 2.8 & $\ldots$ & 2.8 & 2.8 \\
\hline Vertexing $\left(K_{S}^{0}, \Xi^{-}\right)$ & $\ldots$ & $\ldots$ & $\ldots$ & $\ldots$ & $\ldots$ & 5.0 & 5.0 \\
\hline$\Sigma^{0}$ mass & $\ldots$ & $\ldots$ & 1.3 & 1.3 & $\ldots$ & $\ldots$ & $\ldots$ \\
\hline$\Xi^{-}$mass & $\ldots$ & 1.5 & $\ldots$ & $\ldots$ & $\ldots$ & 1.2 & 2.6 \\
\hline$K_{S}^{0}$ mass & $\ldots$ & 0.8 & $\ldots$ & $\ldots$ & $\ldots$ & 1.0 & 1.9 \\
\hline MC modeling & $\ldots$ & 5.4 & $\ldots$ & 5.4 & $\ldots$ & $\ldots$ & $\ldots$ \\
\hline Fitting & 5.9 & 4.7 & 8.0 & 5.0 & 5.2 & 1.4 & 4.1 \\
\hline $\mathcal{B}\left(\Xi^{-} \rightarrow \Lambda \pi^{-}\right)$ & $\ldots$ & $\ldots$ & $\ldots$ & & $\ldots$ & 3.5 & $\ldots$ \\
\hline $\mathcal{B}\left(K_{S}^{0} \rightarrow \pi^{+} \pi^{-}\right)$ & $\ldots$ & $\ldots$ & $\ldots$ & & $\ldots$ & $\ldots$ & 1.0 \\
\hline Total systematic & 7.2 & 8.8 & 8.9 & 9.0 & 5.2 & 8.1 & 9.0 \\
\hline
\end{tabular}

TABLE I. Summary of sources of systematic uncertainties (\%). 
reference decay modes, leading to a systematic uncertainty of less than $1 \%$ obtained by changing the photon energy cut in both modes. The uncertainty due to track finding is about $2.8 \%$ for modes with higher multiplicity than the reference decay mode. The systematic uncertainty due to a $\pi^{ \pm}$ misidentified as a $K^{ \pm}$is below $1 \%$. By studying large signal MC samples the change in detection efficiency with and without a vertex requirement for $\mathrm{K}_{\mathrm{S}}^{0} \rightarrow \pi^{+} \pi^{-}$ and $\Xi^{-} \rightarrow \Lambda \pi^{-}$, we assign a systematic uncertainty due to the lack of vertexing for $K_{S}^{0}$ and $\Xi^{-}$to be $5 \%$ for the modes with such a particle in the final state. We use a simplistic phase space model to generate signal $\mathrm{MC}$ for $\Lambda_{c}^{+} \rightarrow$ $\Lambda K^{+} \pi^{+} \pi^{-}$and $\Lambda_{c}^{+} \rightarrow \Sigma^{0} K^{+} \pi^{+} \pi^{-}$. We assign $5.4 \%$ for signal MC modeling.

We also study possible biases due to our fitting procedure by varying the fitted function as describled below. Conservatively, the effect of all changes to the signal yield are accounted as systematic uncertainty. For each distribution we vary the order of the polynomial background, and vary the signal width $(\sigma)$ by 1 standard deviation. In addition, for $\Lambda_{c}^{+} \rightarrow \Lambda \pi^{+}$we vary all the parameters describing the $\Sigma^{0}$ and $\Xi_{c}$ reflections by 1 standard deviation. The systematic uncertainty due to fit bias is assigned to be $5.9 \%$ for $\mathcal{B}\left(\Lambda_{c}^{+} \rightarrow \Lambda K^{+}\right) / \mathcal{B}\left(\Lambda_{c}^{+} \rightarrow \Lambda \pi^{+}\right), \quad 8 \%$ for $\mathcal{B}\left(\Lambda_{c}^{+} \rightarrow \Sigma^{0} K^{+}\right) / \mathcal{B}\left(\Lambda_{c}^{+} \rightarrow \Sigma^{0} \pi^{+}\right)$, and $4.7 \%$ for $\mathcal{B}\left(\Lambda_{c}^{+} \rightarrow \Lambda K^{+} \pi^{+} \pi^{-}\right) / \mathcal{B}\left(\Lambda_{c}^{+} \rightarrow \Lambda \pi^{+}\right)$. The systematic uncertainty associated with the fitting is found to be the dominant one for the Cabibbo-suppressed decay modes. Published data [1] provide the uncertainty in the daughter branching fractions $(1 \%-4 \%)$

\section{SUMMARY}

We measure the branching ratio of the Cabibbosuppressed decay $\Lambda_{c}^{+} \rightarrow \Lambda K^{+}$relative to the Cabibbo- favored decay mode $\Lambda_{c}^{+} \rightarrow \Lambda \pi^{+}$to be $0.044 \pm$ 0.004 (stat.) \pm 0.003 (syst.), which is somewhat lower and substantially more precise than the previous measurement, $0.074 \pm 0.010$ (stat.) \pm 0.012 (syst.) [3]. We also report the branching ratio of the Cabibbo-suppressed decay $\Lambda_{c}^{+} \rightarrow$ $\Sigma^{0} K^{+}$relative to the Cabibbo-favored decay mode $\Lambda_{c}^{+} \rightarrow$ $\Sigma^{0} \pi^{+}$to be $0.038 \pm 0.005$ (stat.) \pm 0.003 (syst.). It is also lower and substantially more precise than the previous measurement, $0.056 \pm 0.014 \pm 0.003$ [3]. We also report the first searches for the Cabibbo-suppressed decays $\Lambda_{c}^{+} \rightarrow$ $\Lambda K^{+} \pi^{+} \pi^{-}$and $\Lambda_{c}^{+} \rightarrow \Sigma^{0} K^{+} \pi^{+} \pi^{-}$. We do not observe statistically significant signals for these decay modes and therefore set upper limits at the $90 \%$ C.L. The results for the Cabibbo-suppressed decays are shown in Table II. We finally report the branching ratio measurement of the Cabibbo-favored decays $\Lambda_{c}^{+} \rightarrow \Sigma^{0} \pi^{+}, \Lambda_{c}^{+} \rightarrow \Xi^{-} K^{+} \pi^{+}$ and $\Lambda_{c}^{+} \rightarrow \Lambda \overline{\mathrm{K}}^{0} K^{+}$relative to the Cabibbo-favored decay mode $\Lambda_{c}^{+} \rightarrow \Lambda \pi^{+}$as shown in Table III. These results represent a marked improvement on the existing numbers [1] and the results for the two body decays are also in agreement with the predictions for these modes. The expectations from the quark model [4] are $\mathcal{B}\left(\Lambda_{\mathrm{c}}^{+} \rightarrow\right.$ $\left.\Lambda \mathrm{K}^{+}\right) / \mathcal{B}\left(\Lambda_{\mathrm{c}}^{+} \rightarrow \Lambda \pi^{+}\right)=[0.039-0.056]$ and $\mathcal{B}\left(\Lambda_{\mathrm{c}}^{+} \rightarrow\right.$ $\left.\Sigma^{0} \mathrm{~K}^{+}\right) / \mathcal{B}\left(\Lambda_{\mathrm{c}}^{+} \rightarrow \Sigma^{0} \pi^{+}\right)=[0.033-0.036]$.

\section{ACKNOWLEDGMENTS}

We are grateful for the extraordinary contributions of our PEP-II colleagues in achieving the excellent luminosity and machine conditions that have made this work possible. The success of this project also relies critically on the expertise and dedication of the computing organizations that support $B A B A R$. The collaborating institutions wish to thank SLAC for its support and the kind hospitality extended to them. This work is supported by the US

TABLE II. Summary of signal yields, relative efficiencies and branching fraction ratios with respect to the reference mode for the Cabibbo-suppressed decays of $\Lambda_{c}^{+}$, where the first uncertainty is statistical and second one represents the systematic uncertainty. The decay $\Lambda_{\mathrm{c}}^{+} \rightarrow \Lambda \pi^{+}$is the reference mode for $\Lambda_{c}^{+} \rightarrow \Lambda K^{+}$and $\Lambda_{c}^{+} \rightarrow \Lambda K^{+} \pi^{+} \pi^{-}$signal decay modes. The decay $\Lambda_{\mathrm{c}}^{+} \rightarrow \Sigma^{0} \mathrm{~K}^{+}$is the reference mode for $\Lambda_{c}^{+} \rightarrow \Sigma^{0} K^{+}$and $\Lambda_{c}^{+} \rightarrow \Sigma^{0} K^{+} \pi^{+} \pi^{-}$signal decay modes.

\begin{tabular}{lccc}
\hline \hline Signal mode & Signal yield & Relative efficiency & $\mathcal{B}_{\text {signal }} / \mathcal{B}_{\text {reference }}$ \\
\hline$\Lambda K^{+}\left(x_{p}>0.5\right)$ & $1162 \pm 101$ & $0.781 \pm 0.010$ & $0.044 \pm 0.004 \pm 0.003$ \\
$\Lambda K^{+} \pi^{+} \pi^{-}\left(x_{p}>0.6\right)$ & $160 \pm 62$ & $0.310 \pm 0.010$ & $<4.1 \times 10^{-2} 90 \%$ C.L. \\
$\Sigma^{0} K^{+}\left(x_{p}>0.5\right)$ & $366 \pm 52$ & $0.780 \pm 0.010$ & $0.038 \pm 0.005 \pm 0.003$ \\
$\Sigma^{0} K^{+} \pi^{+} \pi^{-}\left(x_{p}>0.6\right)$ & $21 \pm 24$ & $0.390 \pm 0.010$ & $<2.0 \times 10^{-2} 90 \%$ C.L. \\
\hline \hline
\end{tabular}

TABLE III. Summary of signal yields, relative efficiencies and branching fraction ratios with respect to the reference mode for the Cabibbo-favored decays of $\Lambda_{c}^{+}$, where the first uncertainty is statistical and the second one represents the systematic uncertainty. The decay $\Lambda_{\mathrm{c}}^{+} \rightarrow \Lambda \pi^{+}$is the reference mode for $\Lambda_{\mathrm{c}}^{+} \rightarrow \Sigma^{0} \pi^{+}, \Lambda_{\mathrm{c}}^{+} \rightarrow \Xi^{-} \mathrm{K}^{+} \pi^{+}$, and $\Lambda_{\mathrm{c}}^{+} \rightarrow \Lambda \overline{\mathrm{K}}^{0} \mathrm{~K}^{+}$signal decay modes.

\begin{tabular}{lcrr}
\hline \hline Signal mode & Signal yield & Relative efficiency & $\mathcal{B}_{\text {signal }} / \mathcal{B}_{\text {reference }}$ \\
\hline$\Sigma^{0} \pi^{+}\left(x_{p}>0.5\right)$ & $32693 \pm 324$ & $1.013 \pm 0.010$ & $0.977 \pm 0.015 \pm 0.051$ \\
$\Xi^{-} K^{+} \pi^{+}\left(x_{p}>0.6\right)$ & $2665 \pm 84$ & $0.250 \pm 0.003$ & $0.480 \pm 0.016 \pm 0.039$ \\
$\Lambda \bar{K}^{0} K^{+}\left(x_{p}>0.6\right)$ & $460 \pm 30$ & $0.152 \pm 0.020$ & $0.395 \pm 0.026 \pm 0.036$ \\
\hline \hline
\end{tabular}


Department of Energy and National Science Foundation, the Natural Sciences and Engineering Research Council (Canada), Institute of High Energy Physics (China), the Commissariat à l'Energie Atomique and Institut National de Physique Nucléaire et de Physique des Particules (France), the Bundesministerium für Bildung und Forschung and Deutsche Forschungsgemeinschaft (Germany), the Istituto Nazionale di Fisica Nucleare (Italy), the Foundation for Fundamental Research on
Matter (The Netherlands), the Research Council of Norway, the Ministry of Science and Technology of the Russian Federation, and the Particle Physics and Astronomy Research Council (United Kingdom). Individuals have received support from CONACyT (Mexico), the A.P. Sloan Foundation, the Research Corporation, and the Alexander von Humboldt Foundation.
[1] W.-M. Yao et al., J. Phys. G 33, 1 (2006).

[2] J. P. Alexander et al. (CLEO Collaboration), Phys. Rev. D 53, R1013 (1996).

[3] K. Abe et al. (Belle Collaboration), Phys. Lett. B 524, 33 (2002).

[4] T. Uppal, R. C. Verma, and M. P. Khanna, Phys. Rev. D 49, 3417 (1994).

[5] J. G. Körner and M. Krämer, Z. Phys. C 55, 659 (1992).

[6] J. G. Körner, M. Krämer, and J. Willrodt, Z. Phys. C 2, 117
(1979).

[7] B. Aubert et al. (BABAR Collaboration), Nucl. Instrum. Methods Phys. Res., Sect. A 479, 1 (2002).

[8] T. Sjöstrand, Comput. Phys. Commun. 82, 74 (1994).

[9] S. Agostinelli et al. (GEANT4 Collaboration), Nucl. Instrum. Methods Phys. Res., Sect. A 506, 250 (2003).

[10] G. J. Feldman and R. D. Cousins, Phys. Rev. D 57, 3873 (1998). 